\title{
A MULTIPLIER THEOREM FOR ANALYTIC FUNCTIONS OF SLOW MEAN GROWTH
}

\author{
A. MATHESON
}

\begin{abstract}
Multipliers into $l^{q}$ spaces are characterized for certain spaces of functions analytic in the unit disk whose $p$-means $(0<p<1)$ do not grow too rapidly.
\end{abstract}

1. The purpose of this paper is to extend some results on multiplier sequences to certain spaces of analytic functions first studied by Hardy and Littlewood. For an exponent $p(0<p<\infty)$ and a positive number $a$, let $H_{a}^{p}$ denote the class of all functions $f(z)$ analytic in the open unit disk for which

$$
M_{p}(f, r)=O\left((1-r)^{-a}\right), \quad r \rightarrow 1^{-},
$$

where, as usual,

$$
M_{p}(f, r)=\left\{\frac{1}{2 \pi} \int_{-\pi}^{\pi}\left|f\left(r e^{i \theta}\right)\right|^{p} d \theta\right\}^{1 / p} .
$$

The multipliers from $H_{a}^{p}$ to $l^{q}(0<p \leqslant 1)$ will be described in $\S 3$. To do this, an invariant metric will be introduced on $H_{a}^{p}$ in $\$ 2$ and it will be shown that $H_{a}^{p}$ is complete with respect to this metric. It will follow that $H_{a}^{p}$ is an $F$-space, and hence the tools of functional analysis, in particular the closed graph theorem will become available.

If $X$ is a set of functions analytic in the unit disk, then every function $f$ in $X$ has a Taylor series expansion $f(z)=\sum_{n=0}^{\infty} \hat{f}(n) z^{n}$. A sequence $\lambda=\left\{\lambda_{n}\right\}_{n=0}^{\infty}$ of complex numbers is called a multiplier of $X$ into $l^{q}$, written $\lambda \in M\left(X, l^{q}\right)$ if the sequence $\left\{\lambda_{n} \hat{f}(n)\right\}_{n=0}^{\infty}$ belongs to $l^{q}$ for every $f$ in $X$. Multiplier sequences have been studied extensively by many authors. In particular Duren and Shields [1] described the multipliers from $H^{p}$ to $l^{\infty}(0<p<1)$ and $H^{p}$ to $l^{q}$ $(0<p<1, p<q<\infty)$. In $\$ 3$ these results will be extended to describe the multipliers from $H_{a}^{p}$ to $l^{q}$.

The following theorems, essentially due to Hardy and Littlewood [2], will be needed in the sequel.

THEOREM A. If $f$ is analytic in the open unit disk, and $0<p<1$, then

$$
M_{1}(f, r) \leqslant M_{\infty}^{1-t}(f, r) M_{p}^{t}(f, r)
$$

for all $t, 0 \leqslant t \leqslant p$.

Received by the editors August 16, 1978.

AMS (MOS) subject classifications (1970). Primary 30A78.

(C) 1979 American Mathematical Society 0002-9939/79/0000-0462/\$02.25 
THEOREM B. If $f$ is analytic in the open unit disk, $0<p<1, a>0$, and

$$
M_{p}(f, r) \leqslant C(1-r)^{-a}, \quad 0<r<1,
$$

then

$$
M_{\infty}(f, r) \leqslant A C(1-r)^{-a-1 / p}, \quad 0 \leqslant r<1,
$$

where $A$ is a constant depending only on $p$ and $a$.

THEOREM C. If $f, C, p$ and $a$ are as in Theorem $\mathrm{B}$, then

$$
M_{1}(f, r) \leqslant A C(1-r)^{1-a-1 / p}
$$

where $A$ is a constant depending only on $p$ and $a$.

Theorem $\mathbf{A}$ is a simple consequence of Hölder's inequality and Jensen's convexity theorem. Theorem $B$ follows from a nontrivial mean value property of functions $|u|^{p}$ where $u$ is harmonic, and Theorem $\mathrm{C}$ is an immediate consequence of Theorems $\mathrm{A}$ and $\mathrm{B}$.

2. For fixed $p(0<p<\infty)$ and $a(a \geqslant 0)$ define

$$
\|f\|=\|f\|_{p, a}= \begin{cases}\sup _{0<r<1}(1-r)^{a} M_{p}(f, r), & p \geqslant 1, \\ \sup _{0<r<1}(1-r)^{p a} M_{p}^{p}(f, r), & p<1,\end{cases}
$$

for $f$ analytic in the open unit disk. The space $H_{a}^{p}$ consists of those $f$ for which $\|f\|_{p, a}<\infty$. It is clear that $H_{a}^{p}$ is a linear space and that \|\|$_{p, a}$ defines an invariant metric in $H_{a}^{p}$, which is a norm if $p \geqslant 1$. Also $H_{0}^{p}$ is the classical Hardy space $H^{p}$. It will be shown below that $H_{a}^{p}$ is complete, hence an $F$-space $(B$-space if $p \geqslant 1)$. The following lemma will be needed.

LEMMA 1. Let $\psi_{n}(r)$ be a sequence of nonnegative functions on $[0,1)$ satisfying:

(a) $\psi_{n}(r) \leqslant B$ for $n=1,2, \ldots, 0 \leqslant r<1$,

(b) $\lim _{n \rightarrow \infty} \sup _{0<r<1} \psi_{n}(r)$ exists and is finite,

(c) $\lim _{n \rightarrow \infty} \psi_{n}(r)=\psi(r)$ exists for each $r, 0<r<1$;

then

$$
\sup _{0<r<1} \psi(r) \leqslant \lim _{n \rightarrow \infty} \sup _{0<r<1} \psi_{n}(r)
$$

Proof. Let $C=\lim _{n \rightarrow \infty} \sup _{0<r<1} \psi_{n}(r)$ and $D=\sup _{0<r<1} \psi(r)$; clearly $C \leqslant B, D \leqslant B$. Let $\varepsilon>0$. There exists $r_{0}, 0 \leqslant r_{0}<1$ such that

$$
D-\varepsilon / 3<\psi\left(r_{0}\right) \leqslant D
$$

and a positive integer $n_{0}$ such that

$$
\left|\psi_{n}\left(r_{0}\right)-\psi\left(r_{0}\right)\right|<\varepsilon / 3 \text { if } n>n_{0}
$$


thus

$$
\begin{aligned}
\psi\left(r_{0}\right) & <\psi_{n}\left(r_{0}\right)+\varepsilon / 3, \quad n>n_{0}, \\
& \leqslant \sup _{0<r<1} \psi_{n}(r)+\varepsilon / 3, \quad n>n_{0}, \\
& \leqslant \lim _{n \rightarrow \infty} \sup _{0<r<1} \psi_{n}(r)+2 \varepsilon / 3 \\
& =C+2 \varepsilon / 3 .
\end{aligned}
$$

Therefore, $D-\varepsilon / 3 \leqslant C+2 \varepsilon / 3$. The lemma follows on letting $\varepsilon \rightarrow 0$.

Proposition 1. The spaces $H_{a}^{p}, 0<p<\infty, a>0$, are complete.

Proof. Let $\left\{f_{n}\right\}_{n=1}^{\infty}$ be a Cauchy sequence in $H_{a}^{p}$. Defining $f_{n, r}(z)=f_{n}(r z)$, it is easy to see that for each $r<1$ the sequence $\left\{f_{n, r}\right\}_{n=1}^{\infty}$ is a Cauchy sequence in the Hardy space $H^{p}$. In particular the sequence $\left\{f_{n}\right\}_{n=1}^{\infty}$ converges uniformly on compact subsets of the open unit disk. Define $f(z)=$ $\lim _{n \rightarrow \infty} f_{n}(z)$ for $|z|<1$. Clearly $\left\|f_{n}\right\|$ converges to some finite limit, say $\lim _{n \rightarrow \infty}\left\|f_{n}\right\|=B$, and for each $r, 0 \leqslant r<1, M_{p}\left(f-f_{n}, r\right) \rightarrow 0$ as $n \rightarrow \infty$. Hence for $1 \leqslant p, M_{p}(f, r)=\lim _{n \rightarrow \infty} M_{p}\left(f_{n}, r\right)$, so

$$
\begin{aligned}
(1-r)^{a} M_{p}(f, r) & =(1-r)^{a} \lim _{n \rightarrow \infty} M_{p}\left(f_{n}, r\right) \\
& \leqslant \lim _{n \rightarrow \infty}\left\|f_{n}\right\|=B, \quad 0<r<1 .
\end{aligned}
$$

Thus, $\|f\|_{p, a} \leqslant B<\infty$, so $f \in H_{a}^{p}$. If $0<p<1$,

$$
\begin{aligned}
M_{p}^{p}(f, r) & \leqslant \lim _{n \rightarrow \infty} M_{p}^{p}\left(f-f_{n}, r\right)+\lim _{n \rightarrow \infty} M_{p}^{p}\left(f_{n}, r\right) \\
& =\lim _{n \rightarrow \infty} M_{p}^{p}\left(f_{n}, r\right),
\end{aligned}
$$

and as above, $\|f\|_{p, a} \leqslant B<\infty$, so $f \in H_{a}^{p}$. It follows from Lemma 1 applied to the functions $\psi_{n}(r)=(1-r)^{a} M_{p}\left(f_{n}-f_{m}, r\right)$, that for each fixed $m$,

$$
\sup _{0<r<1} \lim _{n \rightarrow \infty}(1-r)^{a} M_{p}\left(f_{n}-f_{m}, r\right) \leqslant \lim _{n \rightarrow \infty} \sup _{0<r<1}(1-r)^{a} M_{p}\left(f_{n}-f_{m}, r\right) \text {. }
$$

Therefore

$$
\begin{aligned}
\left\|f-f_{m}\right\| & =\sup _{0<r<1}(1-r)^{a(p)} M_{p}^{(p)}\left(f-f_{m}, r\right) \\
& =\sup _{0<r<1} \lim _{n \rightarrow \infty}(1-r)^{a(p)} M_{p}^{(p)}\left(f_{n}-f_{m}, r\right) \\
& \leqslant \lim _{n \rightarrow \infty} \sup _{0<r<1}(1-r)^{a(p)} M_{p}^{(p)}\left(f_{n}-f_{m}, r\right) \\
& =\lim _{n \rightarrow \infty}\left\|f_{n}-f_{m}\right\|,
\end{aligned}
$$

since

$$
(1-r)^{a} M_{p}\left(f-f_{m}, r\right)=\lim _{n \rightarrow \infty}(1-r)^{a} M_{p}\left(f_{n}-f_{m}, r\right)
$$

for each $r, 0<r<1$. Here $(p)$ is $p$ or 1 according as $p<1$ or $p>1$. It follows that $\left\|f-f_{m}\right\| \rightarrow 0$ as $m \rightarrow \infty$. This completes the proof. 
3. This section is devoted to describing the multipliers from $H_{a}^{p}(0<p<1)$ to $l^{q}$. The following three lemmas will be used. The first is due to Hardy and Littlewood [2]. Since the proofs are brief, they will be included.

LEMMA 2. If $f \in H_{a}^{p}(0<p \leqslant 1, a>0)$, then

$$
|\hat{f}(n)| \leqslant C\|f\|_{p, a}^{1 / p} n^{a+1 / p-1}, \quad n=1,2, \ldots,
$$

where $C$ is a constant depending only on $p$ and $a$.

Proof. By Cauchy's formula,

$$
|\hat{f}(n)| \leqslant r^{-n} M_{1}(f, r) \leqslant C r^{-n}\|f\|^{1 / p}(1-r)^{1-a-1 / p}
$$

by Theorem $\mathrm{C}$, and the lemma follows on setting $r=1-1 / n$.

LEMMA 3. For $\gamma>0$, let $g_{\gamma}(z)=(1-z)^{-\gamma}$. Then

(a) $\hat{g}_{\gamma}(n) \sim n^{\gamma-1} / \Gamma(\gamma)$,

(b) $g_{\gamma} \in H_{a}^{p}(0<p<\infty, a>0)$ if $1 / p<\gamma<a+1 / p$.

Proof. The first part is well known (apply Stirling's formula to the coefficients in the binomial series). The second part follows from the estimate

$$
\int_{-\pi}^{\pi}\left(1-2 r \cos \theta+r^{2}\right)^{\delta / 2} d \theta=O\left((1-r)^{1-\delta}\right), \quad \delta>1 .
$$

If $p \gamma>1$,

$$
\begin{aligned}
M_{p}^{p}\left(g_{\gamma}, r\right) & =\frac{1}{2 \pi} \int_{-\pi}^{\pi}\left(1-2 r \cos \theta+r^{2}\right)^{-p \gamma / 2} d \theta \\
& =O\left((1-r)^{1-p \gamma}\right)=O\left((1-r)^{-\alpha p}\right] \text { if } a>\gamma-1 / p
\end{aligned}
$$

LEMMA 4. If $\left\|f_{n}-f\right\|_{p, a} \rightarrow 0$, then $\hat{f}_{n}(k) \rightarrow \hat{f}(k)$ for all $k$.

Proof.

$$
\begin{aligned}
\left|\hat{f}_{n}(k)-\hat{f}(k)\right| & =\left|\frac{1}{2 \pi i} \int_{|z|=1 / 2}\left(f_{n}(z)-f(z)\right) / z^{k+1} d z\right| \\
& \leqslant 2^{k} M_{1}\left(f_{n}-f, 1 / 2\right) \leqslant B\left\|f_{n}-f\right\|_{p, a} .
\end{aligned}
$$

The main result describes the multipliers from $H_{a}^{p}(0<p<1)$ to $l^{q}$.

THEOREM 1. The sequence $\lambda=\left\{\lambda_{n}\right\}_{n=0}^{\infty}$ is a multiplier from $H_{a}^{p}$ to $l^{q}$ $(0<p<1, a>0)$ if and only if the sequence $\left\{n^{a+1 / p-1} \lambda_{n}\right\}_{n=0}^{\infty}$ belongs to $l^{q}$.

Proof. Case $q=\infty$. If $\left|\lambda_{n}\right| \leqslant C n^{1-1 / p-a}$ and $f \in H_{a}^{p}$, then by Lemma 2,

$$
\left|\lambda_{n} \hat{f}(n)\right| \leqslant C^{\prime}\|f\| n^{a+1 / p-1} n^{1-a-1 / p}=C^{\prime}\|f\|
$$

and $\lambda$ is a multiplier. Conversely, if $\lambda$ is a multiplier, $\lambda$ defines a linear operator $\Lambda: H_{a}^{p} \rightarrow l^{\infty}$ given by $\Lambda(f)=\left\{\lambda_{n} \hat{f}(n)\right\}_{n=0}^{\infty}$. If $f_{n} \rightarrow f$ in $H_{a}^{p}$ and $\left\{\lambda_{k} \hat{f}_{n}(k)\right\} \rightarrow\left\{s_{k}\right\}$ in $l^{\infty}$, then $s_{k}=\lambda_{k} \hat{f}(k)$ for each $k$, since $\hat{f}_{n}(k) \rightarrow \hat{f}(k)$ for each $k$, by Lemma 4 . Thus $\Lambda$ has closed graph. Hence there exists a constant 
$C$ such that $\left|\lambda_{k} \hat{f}(k)\right|<C\|f\|$ for all $k$ and all $f \in H_{a}^{p}$. Applying this inequality to the function $g_{\gamma}$ with $\gamma=1 / p+a$ proves the theorem.

Case $q<\infty$. As above, if $\left\{\lambda_{n}\right\}_{n=0}^{\infty}$ is a multiplier, then an application of the closed graph theorem gives a constant $C$ such that

$$
\sum_{n=0}^{\infty}\left|\lambda_{n} \hat{f}(n)\right|^{q} \leqslant C\|f\|_{p, a}^{q} .
$$

Applying this inequality to the function $g_{\gamma}, \gamma=1 / p+a$ proves the necessity. The sufficiency is immediate from Lemma 2 . That completes the proof.

Remarks. (1) The use of the closed graph theorem is standard in problems of this sort.

(2) That the condition on multipliers in Theorem 1 is simpler than the condition of Duren and Shields for the Hardy spaces $H^{p}$ and for $q<\infty$, follows from the fact that $(1-z)^{-1}$ does not belong to $H^{1}$, but belongs to $H_{a}^{1}$ for all $a>0$. The more elementary proof here follows for the same reason.

\section{BIBLIOGRAPHY}

1. P. L. Duren and A. L. Shields, Properties of $H^{p}(0<p<1)$ and its containing B-space, Trans. Amer. Math. Soc. 141 (1969), 255-262.

2. G. H. Hardy and J. E. Littlewood, Some properties of conjugate functions, J. Reine Angew. Math. 167 (1931), 405-423.

Department of Mathematics, OkLahoma State University, Stillwater, OkLahoma 74074 